Preprint RM3-TH/99-2

\title{
Relativistic effects on the neutron charge form factor in the constituent quark model
}

\author{
Fabio Cardarelli and Silvano Simula \\ Istituto Nazionale di Fisica Nucleare, Sezione Roma III, \\ Via della Vasca Navale 84, I-00146 Roma, Italy
}

\begin{abstract}
The neutron charge form factor $G_{E}^{n}\left(Q^{2}\right)$ is investigated within a constituent quark model formulated on the light-front. It is shown that, if the quark initial motion is neglected in the Melosh rotations, the Dirac neutron form factor $F_{1}^{n}\left(Q^{2}\right)$ receives a relativistic correction which cancels exactly against the Foldy term in $G_{E}^{n}\left(Q^{2}\right)$, as it has been recently argued by Isgur. Moreover, at the same level of approximation the ratio of the proton to neutron magnetic form factors $G_{M}^{p}\left(Q^{2}\right) / G_{M}^{n}\left(Q^{2}\right)$ is still given by the naive $S U(6)$-symmetry expectation, $-3 / 2$. However, it is also shown that the full Melosh rotations break $S U(6)$ symmetry, giving rise to $G_{E}^{n}\left(Q^{2}\right) \neq 0$ and $G_{M}^{p}\left(Q^{2}\right) / G_{M}^{n}\left(Q^{2}\right) \neq-3 / 2$ even when a $S U(6)$-symmetric canonical wave function is assumed. It turns out that relativistic effects alone cannot explain simultaneously the experimental data on $G_{E}^{n}\left(Q^{2}\right)$ and $G_{M}^{p}\left(Q^{2}\right) / G_{M}^{n}\left(Q^{2}\right)$.
\end{abstract}

\footnotetext{
${ }^{a}$ To appear in Physics Letters B.
} 
The elastic nucleon form factors contain important pieces of information on the internal structure of the nucleon and therfore an extensive program aimed at their experimental investigation is currently undergoing and planned at several existing facilities around the world [1]. In what follows we will focus on the so-called nucleon Sachs form factors, which are defined as [2]

$$
\begin{aligned}
G_{E}^{N}\left(Q^{2}\right) & =F_{1}^{N}\left(Q^{2}\right)-\frac{Q^{2}}{4 M^{2}} F_{2}^{N}\left(Q^{2}\right) \\
G_{M}^{N}\left(Q^{2}\right) & =F_{1}^{N}\left(Q^{2}\right)+F_{2}^{N}\left(Q^{2}\right)
\end{aligned}
$$

where $F_{1}^{N}\left(Q^{2}\right)\left[F_{2}^{N}\left(Q^{2}\right)\right]$ is the Dirac [Pauli] nucleon form factor, appearing in the usual covariant decomposition of the nucleon electromagnetic current matrix elements, viz.

$$
<N\left(p^{\prime}, s^{\prime}\right)\left|j_{e m}^{\mu}(0)\right| N(p, s)>=\bar{u}\left(p^{\prime}, s^{\prime}\right)\left\{F_{1}^{N}\left(Q^{2}\right) \gamma^{\mu}+F_{2}^{N}\left(Q^{2}\right) \frac{i \sigma^{\mu \nu} q_{\nu}}{2 M}\right\} u(p, s)
$$

with $Q^{2}=-q \cdot q$ and $M$ being the squared four-momentum transfer and the nucleon mass, respectively. As it is well known, the nucleon Sachs form factors may be interpreted in the Breit frame as the Fourier transforms of the nucleon charge and magnetisation density, respectively. In this respect, from Eq. (11) the squared nucleon charge radius, $r_{N}^{2}$, is therefore given by the sum of two terms, namely

$$
r_{N}^{2} \equiv-6\left[\frac{d G_{E}^{N}\left(Q^{2}\right)}{d Q^{2}}\right]_{Q^{2}=0}=r_{1 N}^{2}+\frac{3 k_{N}}{2 M^{2}}
$$

where $r_{1 N}^{2} \equiv-6\left[d F_{1}^{N}\left(Q^{2}\right) / d Q^{2}\right]_{Q^{2}=0}$ and $k_{N} \equiv F_{2}^{N}(0)$ is the nucleon anomalous magnetic moment. The second term in the r.h.s of Eq. (3) is usually referred to as the Foldy contribution and it is of relativistic origin.

In case of the neutron the charge radius has been nicely determined from electronneutron elastic scattering experiments at very low energy, obtaining $r_{n}^{2}=-0.113 \pm 0.005 \mathrm{fm}^{2}$ [3]. Since from Eq. (3) the Foldy contribution turns out to be $\simeq-0.126 \mathrm{fm}^{2}$, the experimental value of the neutron charge radius appears to be almost totally explained by its Foldy term alone, i.e. by relativistic effects. This result, which implies a small value for

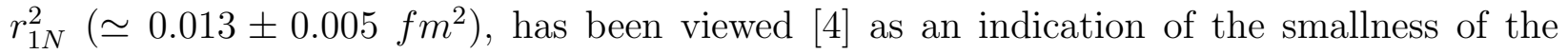
intrinsic charge radius related to the neutron rest-frame charge distribution. Nevertheless, very recently [0] the interpretation of the neutron charge radius as arising from its internal charge distribution has been asserted again. Indeed, it has been argued that, going beyond the non-relativistic limit when the Foldy term firstly appears, the Dirac neutron form factor $F_{1}^{n}\left(Q^{2}\right)$ receives a relativistic correction that cancels exactly against the Foldy term in $G_{E}^{n}\left(Q^{2}\right)$. Such a statement has been inferred from the observation that the well-known phenomenon of zitterbewegung, which produces the Foldy term, cannot contribute to the charge radius of the neutron, because the latter has zero total charge [5].

The aim of this letter is to address the issue of the relativistic effects on $G_{E}^{n}\left(Q^{2}\right)$ adopting a constituent quark $(C Q)$ model formulated on the light-front. 
Let us briefly recall the basic notation and the relevant structure of the nucleon wave function in the light-front formalism. Following, e.g., Refs. [6, 7, 8] the light-front nucleon wave function is eigenstate of the non-interacting angular momentum operators $j^{2}$ and $j_{n}$, where the vector $\hat{n}=(0,0,1)$ defines the spin quantization axis. For a system of three quarks with equal masses the squared free-mass operator is given by $M_{0}^{2}=\sum_{i=1}^{3}\left(k_{i \perp}^{2}+m^{2}\right) / \xi_{i}$, where $m$ is the $C Q$ mass, $\xi_{i}=p_{i}^{+} / P^{+}$and $\vec{k}_{i \perp}=\vec{p}_{i \perp}-\xi_{i} \vec{P}_{\perp}$ are the intrinsic light-front variables. The subscript $\perp$ indicates the projection perpendicular to the spin quantization axis and the plus component of a 4-vector $p \equiv\left(p^{0}, \vec{p}\right)$ is given by $p^{+}=p^{0}+\hat{n} \cdot \vec{p}$; finally $\tilde{P} \equiv\left(P^{+}, \vec{P}_{\perp}\right)=$ $\tilde{p}_{1}+\tilde{p}_{2}+\tilde{p}_{3}$ is the light-front nucleon momentum and $\tilde{p}_{i}$ the quark one. In terms of the longitudinal momentum $k_{i n}$, related to the variable $\xi_{i}$ by $k_{i n}=\left[\xi_{i} M_{0}-\left(k_{i \perp}^{2}+m^{2}\right) / \xi_{i} M_{0}\right] / 2$, the free mass operator acquires a familiar form, viz. $M_{0}=\sum_{i=1}^{3} \sqrt{m^{2}+k_{i}^{2}}=\sum_{i=1}^{3} E_{i}$ with $\vec{k}_{i} \equiv\left(\vec{k}_{i \perp}, k_{i n}\right)$. Disregarding the colour degrees of freedom, the light-front nucleon wave function can be written as

$$
\left\langle\left\{\xi_{i} \vec{k}_{i \perp} ; \nu_{i}^{\prime} \tau_{i}\right\} \mid \Psi_{N}^{\nu_{N}}\right\rangle=\sqrt{\frac{E_{1} E_{2} E_{3}}{M_{0} \xi_{1} \xi_{2} \xi_{3}}} \sum_{\left\{\nu_{i}\right\}}\left\langle\left\{\nu_{i}^{\prime}\right\}\left|\mathcal{R}^{\dagger}\right|\left\{\nu_{i}\right\}\right\rangle\left\langle\left\{\overrightarrow{k_{i}} ; \nu_{i} \tau_{i}\right\} \mid \chi_{N}^{\nu_{N}}\right\rangle
$$

where the curly braces \{\} mean a list of indices corresponding to $i=1,2,3 ; \nu_{i}\left(\tau_{i}\right)$ is the third component of the quark spin (isospin); $\mathcal{R}^{\dagger}=\prod_{j=1}^{3} R_{j}^{\dagger}\left(\vec{k}_{j \perp}, \xi_{j}, m\right)$ is the product of individual (generalised) Melosh rotations, viz.

$$
R_{j}\left(\vec{k}_{j \perp}, \xi_{j}, m\right) \equiv \frac{m+\xi_{j} M_{0}-i \vec{\sigma}^{(j)} \cdot\left(\hat{n} \times \vec{k}_{j \perp}\right)}{\sqrt{\left(m+\xi_{j} M_{0}\right)^{2}+k_{j \perp}^{2}}}
$$

with $\vec{\sigma}$ being the ordinary Pauli spin matrices. In what follows we will limit ourselves to the case of a pure $S U(6)$ symmetric canonical (or equal-time) wave function, namely

$$
\left\langle\left\{\vec{k}_{i} ; \nu_{i} \tau_{i}\right\} \mid \chi_{N}^{\nu_{N}}\right\rangle=w_{S}(\vec{k}, \vec{p}) \frac{1}{\sqrt{2}}\left[\Phi_{\nu_{N} \tau_{N}}^{00}\left(\left\{\nu_{i} \tau_{i}\right\}\right)+\Phi_{\nu_{N} \tau_{N}}^{11}\left(\left\{\nu_{i} \tau_{i}\right\}\right)\right]
$$

where $\vec{k}=\left(\vec{k}_{1}-\vec{k}_{2}\right) / 2$ and $\vec{p}=\vec{k}_{3}$ are the Jacobian internal co-ordinates for the threequark system, and $w_{S}(\vec{k}, \vec{p})$ is a completely symmetric radial $S$-wave function. Finally, the spin-isospin function $\Phi_{\nu_{N} \tau_{N}}^{S_{12} T_{12}}\left(\left\{\nu_{i} \tau_{i}\right\}\right)$ is defined as

$$
\begin{aligned}
\Phi_{\nu_{N} \tau_{N}}^{S_{12} T_{12}}\left(\left\{\nu_{i} \tau_{i}\right\}\right)= & \sum_{M_{S}}\left\langle\frac{1}{2} \nu_{1} \frac{1}{2} \nu_{2} \mid S_{12} M_{S}\right\rangle\left\langle S_{12} M_{S} \frac{1}{2} \nu_{3} \mid \frac{1}{2} \nu_{N}\right\rangle . \\
& \sum_{M_{T}}\left\langle\frac{1}{2} \tau_{1} \frac{1}{2} \tau_{2} \mid T_{12} M_{T}\right\rangle\left\langle T_{12} M_{T} \frac{1}{2} \tau_{3} \mid \frac{1}{2} \tau_{N}\right\rangle
\end{aligned}
$$

where $S_{12}\left(T_{12}\right)$ is the total spin (isospin) of the quark pair $(1,2)$. The normalisation of the wave function (6) is: $\sum_{\left\{\nu_{i} \tau_{i}\right\}} \int d \vec{k}_{1} d \vec{k}_{2} d \vec{k}_{3} \delta\left(\vec{k}_{1}+\vec{k}_{2}+\vec{k}_{3}\right)\left|\left\langle\left\{\vec{k}_{i}, \nu_{i} \tau_{i}\right\} \mid \chi_{N}^{\nu_{N}}\right\rangle\right|^{2}=\int d \vec{k} d \vec{p}\left|w_{S}(\vec{k}, \vec{p})\right|^{2}$ $=1$. 
As in Refs. [0, 8] we consider the one-body component of the electromagnetic (e.m.) current operator including $C Q$ form factors, namely

$$
\mathcal{I}^{\nu}=\sum_{j=1}^{3} I_{j}^{\nu}=\sum_{j=1}^{3}\left[e_{j} \gamma^{\nu} f_{1}^{j}\left(Q^{2}\right)+i \kappa_{j} \frac{\sigma^{\nu \mu} q_{\mu}}{2 m} f_{2}^{j}\left(Q^{2}\right)\right]
$$

where $\sigma^{\nu \mu}=\frac{i}{2}\left[\gamma^{\nu}, \gamma^{\mu}\right], e_{j}$ is the charge of the $\mathrm{j}$-th quark, $\kappa_{j}$ the corresponding anomalous magnetic moment and $f_{1(2)}^{j}\left(Q^{2}\right)$ its Dirac (Pauli) form factor (with $f_{1(2)}^{j}(0)=1$ ). It is well known that in the light-front formalism the form factors for a conserved current can be obtained using only the matrix elements of the plus component of the current operator (see, e.g., Ref. [6]) and, moreover, for $Q^{2} \geq 0$ the choice of a frame where $q^{+}=0$ allows to suppress the contribution of the $Z$-graph (i.e., pair creation from the vacuum) [9]. More precisely, in what follows the four-momentum transfer $q$ is taken to be purely transverse, i.e. $q=\left(0, \vec{q}_{\perp}, 0\right)$ with $Q^{2}=-q^{2}=\left|\vec{q}_{\perp}\right|^{2}$. In case of the nucleon one has

$$
\left\langle\Psi_{N}^{\nu_{N}^{\prime}}\left|\mathcal{I}^{+}\right| \Psi_{N}^{\nu_{N}}\right\rangle=F_{1}^{N}\left(Q^{2}\right) \delta_{\nu^{\prime}{ }_{N} \nu_{N}}-i \frac{Q}{2 M} F_{2}^{N}\left(Q^{2}\right)\left\langle\nu_{N}^{\prime}\left|\sigma_{y}\right| \nu_{N}\right\rangle
$$

and therefore $F_{1}^{N}\left(Q^{2}\right)$ is related to the non spin-flip matrix elements, while $F_{2}^{N}\left(Q^{2}\right)$ can be extracted from the spin-flip ones. Using Eqs. (4) the nucleon Dirac and Pauli form factors are explicitly given by 10

$$
\begin{aligned}
& F_{1}^{p(n)}\left(Q^{2}\right)=\frac{3}{2} \int[d \xi]\left[d \vec{k}_{\perp}\right]\left[d \overrightarrow{k^{\prime}}+\right] \sqrt{\frac{E_{1} E_{2} E_{3} M_{0}^{\prime}}{E_{1}^{\prime} E_{2}^{\prime} E^{\prime}{ }_{3} M_{0}}} w_{S}^{*}\left(\overrightarrow{k^{\prime}}, \overrightarrow{p^{\prime}}\right) w_{S}(\vec{k}, \vec{p})\left(\prod_{j=1}^{3} \mathcal{N}_{j}^{\prime} \mathcal{N}_{j}\right) . \\
& \left\{\left[A_{1} A_{2}+\vec{B}_{1} \cdot \vec{B}_{2}\right]\left[A_{3} e_{u(d)} f_{1}^{u(d)}\left(Q^{2}\right)+\tilde{A}_{3} \frac{Q}{2 m} \kappa_{u(d)} f_{2}^{u(d)}\left(Q^{2}\right)\right]+\right. \\
& \frac{1}{9}\left[\left(3 A_{1} A_{2}-\vec{B}_{1} \cdot \vec{B}_{2}\right) A_{3}+2\left(A_{1} \vec{B}_{2}+A_{2} \vec{B}_{1}\right) \cdot \vec{B}_{3}\right] . \\
& {\left[e_{u(d)} f_{1}^{u(d)}\left(Q^{2}\right)+2 e_{d(u)} f_{1}^{d(u)}\left(Q^{2}\right)\right]+\frac{1}{9}\left[\left(3 A_{1} A_{2}-\vec{B}_{1} \cdot \vec{B}_{2}\right) \tilde{A}_{3}+\right.} \\
& \left.\left.2\left(A_{1} \vec{B}_{2}+A_{2} \vec{B}_{1}\right) \cdot \overrightarrow{\tilde{B}}_{3}\right] \frac{Q}{2 m}\left[\kappa_{u(d)} f_{2}^{u(d)}\left(Q^{2}\right)+2 \kappa_{d(u)} f_{2}^{d(u)}\left(Q^{2}\right)\right]\right\} \\
& F_{2}^{p(n)}\left(Q^{2}\right)=-\frac{3 M}{Q} \int[d \xi]\left[d \vec{k}_{\perp}\right]\left[d \overrightarrow{k^{\prime}} \perp\right] \sqrt{\frac{E_{1} E_{2} E_{3} M^{\prime}{ }_{0}}{E^{\prime}{ }_{1} E^{\prime}{ }_{2} E^{\prime}{ }_{3} M_{0}}} w_{S}^{*}\left(\overrightarrow{k^{\prime}}, \overrightarrow{p^{\prime}}\right) w_{S}(\vec{k}, \vec{p})\left(\prod_{j=1}^{3} \mathcal{N}_{j}^{\prime} \mathcal{N}_{j}\right) . \\
& \left\{\left[A_{1} A_{2}+\vec{B}_{1} \cdot \vec{B}_{2}\right] \quad\left[B_{3 y} e_{u(d)} f_{1}^{u(d)}\left(Q^{2}\right)+\tilde{B}_{3 y} \frac{Q}{2 m} \kappa_{u(d)} f_{2}^{u(d)}\left(Q^{2}\right)\right]+\right. \\
& \frac{1}{9}\left[2\left(B_{1 y} \vec{B}_{2}+B_{2 y} \vec{B}_{1}\right) \cdot \vec{B}_{3}-B_{3 y}\left(A_{1} A_{2}+\vec{B}_{1} \cdot \vec{B}_{2}\right)+\right. \\
& \left.2 A_{3}\left(A_{1} B_{2 y}+A_{2} B_{1 y}\right)\right]\left[e_{u(d)} f_{1}^{u(d)}\left(Q^{2}\right)+2 e_{d(u)} f_{1}^{d(u)}\left(Q^{2}\right)\right]+ \\
& \frac{1}{9}\left[2\left(B_{1 y} \vec{B}_{2}+B_{2 y} \vec{B}_{1}\right) \cdot \overrightarrow{\tilde{B}}_{3}-\tilde{B}_{3 y}\left(A_{1} A_{2}+\vec{B}_{1} \cdot \vec{B}_{2}\right)+\right. \\
& \left.\left.2 \tilde{A}_{3}\left(A_{1} B_{2 y}+A_{2} B_{1 y}\right)\right] \frac{Q}{2 m}\left[\kappa_{u(d)} f_{2}^{u(d)}\left(Q^{2}\right)+2 \kappa_{d(u)} f_{2}^{d(u)}\left(Q^{2}\right)\right]\right\}
\end{aligned}
$$


where

$$
\begin{aligned}
{[d \xi]=} & d \xi_{1} d \xi_{2} d \xi_{3} \delta\left[\xi_{1}+\xi_{2}+\xi_{3}-1\right] \\
{\left[d \vec{k}_{\perp}\right]=} & d \vec{k}_{1 \perp} d \vec{k}_{2 \perp} d \vec{k}_{3 \perp} \delta\left[\vec{k}_{1 \perp}+\vec{k}_{2 \perp}+\vec{k}_{3 \perp}\right] \\
{\left[d{\overrightarrow{k^{\prime}}}_{\perp}\right]=} & d{\overrightarrow{k^{\prime}}}_{1 \perp} d{\overrightarrow{k^{\prime}}}_{2 \perp} d{\overrightarrow{k^{\prime}}}_{3 \perp} \delta\left[{\overrightarrow{k^{\prime}}}_{1 \perp}-\vec{k}_{1 \perp}+\xi_{1} \vec{q}_{\perp}\right] \delta\left[{\overrightarrow{k^{\prime}}}_{2 \perp}-\vec{k}_{2 \perp}+\xi_{2} \vec{q}_{\perp}\right] \\
& \delta\left[\vec{k}_{3 \perp}-\vec{k}_{3 \perp}+\left(\xi_{3}-1\right) \vec{q}_{\perp}\right]
\end{aligned}
$$

and

$$
\begin{aligned}
\mathcal{N}_{j} & =1 / \sqrt{\left(m+\xi_{j} M_{0}\right)^{2}+k_{j \perp}^{2}} \\
\mathcal{N}^{\prime}{ }_{j} & =1 / \sqrt{\left(m+\xi_{j} M^{\prime}{ }_{0}\right)^{2}+{k^{\prime}}_{j \perp}^{2}} \\
A_{j} & =\left(m+\xi_{j} M^{\prime}{ }_{0}\right)\left(m+\xi_{j} M_{0}\right)+\vec{k}_{j \perp}^{\prime} \cdot \vec{k}_{j \perp} \\
\tilde{A}_{3} & =\left(m+\xi_{3} M_{0}^{\prime}\right) k_{3 x}-\left(m+\xi_{3} M_{0}\right) k_{3 x}^{\prime} \\
B_{j x} & =\left(m+\xi_{j} M_{0}\right) k_{j y}^{\prime}-\left(m+\xi_{j} M_{0}^{\prime}\right) k_{j y} \\
B_{j y} & =\left(m+\xi_{j} M_{0}^{\prime}\right) k_{j x}-\left(m+\xi_{j} M_{0}\right) k_{j x}^{\prime} \\
B_{j z} & =k_{j x}^{\prime} k_{j y}-k_{j y}^{\prime} k_{j x} \\
\tilde{B}_{3 x} & =k^{\prime}{ }_{3 x} k_{3 y}+k_{3 y}^{\prime} k_{3 x} \\
\tilde{B}_{3 y} & =-\left(m+\xi_{3} M_{0}^{\prime}\right)\left(m+\xi_{3} M_{0}\right)-k_{3 x}^{\prime} k_{3 x}+k_{3 y}^{\prime} k_{3 y} \\
\tilde{B}_{3 z} & =\left(m+\xi_{3} M_{0}^{\prime}\right) k_{3 y}+\left(m+\xi_{3} M_{0}\right) k_{3 y}^{\prime}
\end{aligned}
$$

In the non-relativistic limit, assuming both point-like constituents and the SU(6)symmetric wave function (6), the Fourier transform of the non-relativistic charge density, $\sum_{j=1}^{3} e_{j} \delta\left(\vec{r}-\overrightarrow{r_{j}}\right)$, yields $G_{E}^{N}\left(Q^{2}\right) \rightarrow \sum_{j=1}^{3} e_{j} F_{c}\left(Q^{2}\right)$, i.e. $G_{E}^{p}\left(Q^{2}\right) \rightarrow F_{c}\left(Q^{2}\right)$ and $G_{E}^{n}\left(Q^{2}\right) \rightarrow 0$ with

$$
F_{c}\left(Q^{2}=|\vec{q}|^{2}\right) \equiv \int d \vec{k} d \vec{p} w_{S}^{*}\left(\vec{k}, \vec{p}+\frac{2}{3} \vec{q}\right) w_{S}(\vec{k}, \vec{p})
$$

In a similar way, in case of the non-relativistic magnetisation density the nucleon magnetic form factors are given by $G_{M}^{p}\left(Q^{2}\right) \rightarrow 3 F_{c}\left(Q^{2}\right)$ and $G_{M}^{n}\left(Q^{2}\right) \rightarrow-2 F_{c}\left(Q^{2}\right)$. Note that the $S U(6)$ symmetry predicts both $G_{E}^{n}\left(Q^{2}\right)=0$ and $G_{M}^{p}\left(Q^{2}\right) / G_{M}^{n}\left(Q^{2}\right)=-3 / 2$.

To reach the non-relativistic limit from Eqs. (10-11) is not a trivial task; moreover, as argued in Ref. [5], a subtle cancellation of relativistic corrections is expected to hold when the Foldy term firstly appears. We now want to find out the assumptions that allow to obtain the specific cancellation claimed in Ref. [5] and, then, we want to carry out the appropriate non-relativistic reduction of Eqs. (10-11). The basic assumption made in Ref. [5] is to neglect the transverse motion of the constituents in order to avoid spin-flip effects from the Wigner rotations of the constituent spins. Thus, our first assumption is to put $\vec{k}_{j \perp}=0$ in the Melosh rotations (5), yielding for $j=1,2,3$

$$
R_{j}\left(\vec{k}_{j \perp}, \xi_{j}, m\right) \rightarrow 1
$$


As for the Melosh rotations in the final state (i.e., after virtual photon absorption), we cannot make the same assumption (15), otherwise in case of point-like constituents only a vanishing $F_{2}^{N}\left(Q^{2}\right)$ could be obtained [. Thus, inspired by Ref. [6], we consider the following approximation for $j=1,2,3$

$$
R_{j}\left({\overrightarrow{k^{\prime}}}_{j \perp}, \xi_{j}, m\right) \rightarrow \frac{2 m-i \vec{\sigma}^{(j)} \cdot\left(\hat{n} \times{\overrightarrow{k^{\prime}}}_{j \perp}\right)}{\sqrt{4 m^{2}+{k^{\prime}}^{2}}}
$$

where the denominator is included to maintain the correct normalisation of the final lightfront wave function. Note that the term proportional to $\vec{\sigma}^{(j)}$ in the r.h.s. of Eq. (16) is clearly reminiscent of the non-relativistic magnetisation current. Finally, we have to specify the final transverse momenta $\vec{k}_{j \perp}$ appearing in Eq. (16). To this end we neglect the initial longitudinal motion, i.e. we consider $k_{j n}=0$, which together with the assumption $\vec{k}_{j \perp}=0$ yields $\xi_{j}=1 / 3$. Thus, from the delta functions in Eq. (12) the final transverse momenta turn out to be: ${\overrightarrow{k^{\prime}}}_{1 \perp}={\overrightarrow{k^{\prime}}}_{2 \perp}=-\vec{q}_{\perp} / 3$ and ${\overrightarrow{k^{\prime}}}_{3 \perp}=2 \vec{q}_{\perp} / 3$. Our assumptions $(15-16)$ correspond to consider $\xi_{j} M_{0}=\xi_{j} M_{0}^{\prime}=m$ in Eq. (13), leading to: $\mathcal{N}_{j}=1 / 2 m, \mathcal{N}^{\prime}{ }_{1}=\mathcal{N}^{\prime}{ }_{2}=$ $1 / \sqrt{4 m^{2}+Q^{2} / 9}, \mathcal{N}^{\prime}{ }_{3}=1 / \sqrt{4 m^{2}+4 Q^{2} / 9}, A_{j}=4 m^{2}, \tilde{A}_{3}=2 m Q / 3, B_{1 y}=B_{2 y}=2 m Q / 3$, $B_{3 y}=-4 m Q / 3, \tilde{B}_{3 y}=-4 m^{2}$, while all the other $B$ 's and $\tilde{B}$ 's are identically vanishing. Finally, for compatibility with the non-relativistic reductions $G_{M}^{p}(0) \rightarrow 3$ and $G_{M}^{n}(0) \rightarrow-2$ we neglect any binding effect in the nucleon mass, i.e. we consider $M=3 m$ (cf. also Refs. [5, 6]).

Before carrying out the effects of the assumptions (15-16) on Eqs. (10-11), we have to restrict ourselves to the case of $S U(2)$-symmetric $C Q$ form factors, otherwise any deviation of $G_{E}^{n}\left(Q^{2}\right)$ from zero could be attributed to a possible flavour-dependence of the $C Q$ internal composite structure. Thus, in what follows we will consider

$$
\begin{aligned}
f_{1}^{q}\left(Q^{2}\right) & =f\left(Q^{2}\right) \\
k_{q} f_{2}^{q}\left(Q^{2}\right) & =e_{q} \kappa \tilde{f}\left(Q^{2}\right)
\end{aligned}
$$

where $f\left(Q^{2}\right), \tilde{f}\left(Q^{2}\right)$ and $\kappa$ do not depend on the flavour of the $C Q$ (and $\left.f(0)=\tilde{f}(0)=1\right)$. Using all the above-discussed assumptions it is straightforward to obtain from Eqs. (10-11) the following explicit expressions

$$
\begin{aligned}
& F_{1}^{p}\left(Q^{2}\right)=F_{0}\left(Q^{2}\right)+3 \kappa \frac{Q^{2}}{4 M^{2}} \tilde{F}_{0}\left(Q^{2}\right) \\
& F_{1}^{n}\left(Q^{2}\right)=-2 F_{0}\left(Q^{2}\right) \frac{Q^{2}}{4 M^{2}+Q^{2}}-2 \kappa \tilde{F}_{0}\left(Q^{2}\right) \frac{Q^{2}}{4 M^{2}} \\
& F_{2}^{p}\left(Q^{2}\right)=2 F_{0}\left(Q^{2}\right)+3 \kappa \tilde{F}_{0}\left(Q^{2}\right) \\
& F_{2}^{n}\left(Q^{2}\right)=-2 F_{0}\left(Q^{2}\right) \frac{4 M^{2}}{4 M^{2}+Q^{2}}-2 \kappa \tilde{F}_{0}\left(Q^{2}\right)
\end{aligned}
$$

\footnotetext{
${ }^{b} \mathrm{~A}$ vanishing $F_{2}^{N}\left(Q^{2}\right)$ is appropriate in the case of the heavy-quark limit $m \rightarrow \infty$ (cf. Ref. [11]).
} 
where

$$
F_{0}\left(Q^{2}\right) \equiv \frac{f\left(Q^{2}\right)}{\sqrt{1+Q^{2} / M^{2}}} \int[d \xi]\left[d \vec{k}_{\perp}\right]\left[d \overrightarrow{k^{\prime}} \perp\right] \sqrt{\frac{E_{1} E_{2} E_{3} M_{0}^{\prime}}{E^{\prime}{ }_{1} E_{2}^{\prime} E^{\prime}{ }_{3} M_{0}}} w_{S}^{*}\left(\overrightarrow{k^{\prime}}, \overrightarrow{p^{\prime}}\right) w_{S}(\vec{k}, \vec{p})
$$

while $\tilde{F}_{0}\left(Q^{2}\right)$ is given by Eq. (19) but with $f\left(Q^{2}\right)$ replaced by $\tilde{f}\left(Q^{2}\right)$. In terms of the Sachs form factors (1) one gets

$$
\begin{aligned}
G_{E}^{p}\left(Q^{2}\right) & =\left(1-\frac{Q^{2}}{2 M^{2}}\right) F_{0}\left(Q^{2}\right) \\
G_{E}^{n}\left(Q^{2}\right) & =0 \\
G_{M}^{p}\left(Q^{2}\right) & =3\left[F_{0}\left(Q^{2}\right)+\kappa\left(1+\frac{Q^{2}}{4 M^{2}}\right) \tilde{F}_{0}\left(Q^{2}\right)\right] \\
G_{M}^{n}\left(Q^{2}\right) & =-2\left[F_{0}\left(Q^{2}\right)+\kappa\left(1+\frac{Q^{2}}{4 M^{2}}\right) \tilde{F}_{0}\left(Q^{2}\right)\right]
\end{aligned}
$$

The relativistic corrections to $F_{1,2}^{N}\left(Q^{2}\right)$, contained in Eq. (18), largely differ for proton and neutron, but: i) $F_{1}^{n}\left(Q^{2}\right)$ receives a relativistic correction that cancels exactly against the Foldy term, $-F_{2}^{n}\left(Q^{2}\right) \cdot Q^{2} / 4 M^{2}$, so that the $S U(6)$-symmetry prediction $G_{E}^{n}\left(Q^{2}\right)=0$ still holds, as argued in Ref. [5]; the same type of cancellation does not occur in case of the proton charge form factor $G_{E}^{p}\left(Q^{2}\right)$; ii) the ratio $G_{M}^{p}\left(Q^{2}\right) / G_{M}^{n}\left(Q^{2}\right)$ is still given by the simple $S U(6)$-symmetry expectation, $-3 / 2$. Both results are independent of any particular choice of the $C Q$ form factors (both Dirac and Pauli ones), provided the latter are taken to be $S U(2)$ symmetric (see Eq. (17)). Note that Eq. (20) predicts a vanishing value for $G_{E}^{p}\left(Q^{2}\right)$ at $Q^{2}=2 M^{2}$; this is completely at variance with experimental data and signals that the applicability of Eqs. (18) and (20) is limited only to low values of $Q^{2}$ (i.e., $Q^{2}<<M^{2}$ ). Finally, note also that in Ref. [6] it is claimed that at first order in $Q^{2} / M^{2}$ the leading term in $G_{E}^{n}\left(Q^{2}\right)$ is given by its Foldy term. Such a statement is incorrect, because it is obtained using directly in $G_{E}^{n}\left(Q^{2}\right)$ the non-relativistic limits of $F_{1}^{n}$ and $F_{2}^{n}\left(Q^{2}\right)$, ignoring in this way the Isgur's cancellation mechanism of the Foldy term.

We can now carry out the non-relativistic limit of the nucleon form factors by considering the formal limit $M \rightarrow \infty$ in Eqs. (18) and (20). Taking also into account that the non-relativistic reduction of the integral in Eq. (19) leads to Eq. (14) (see Ref. [6]), one has

$$
\begin{aligned}
& F_{1}^{p}\left(Q^{2}\right) \rightarrow F_{c}\left(Q^{2}\right) f\left(Q^{2}\right) \\
& F_{1}^{n}\left(Q^{2}\right) \rightarrow 0 \\
& F_{2}^{p}\left(Q^{2}\right) \rightarrow F_{c}\left(Q^{2}\right)\left[2 f\left(Q^{2}\right)+3 \kappa \tilde{f}\left(Q^{2}\right)\right] \\
& F_{2}^{n}\left(Q^{2}\right) \rightarrow-2 F_{c}\left(Q^{2}\right)\left[f\left(Q^{2}\right)+\kappa \tilde{f}\left(Q^{2}\right)\right]
\end{aligned}
$$

and

$$
G_{E}^{p}\left(Q^{2}\right) \rightarrow F_{c}\left(Q^{2}\right) f\left(Q^{2}\right)
$$




$$
\begin{aligned}
G_{E}^{n}\left(Q^{2}\right) & \rightarrow 0 \\
G_{M}^{p}\left(Q^{2}\right) & \rightarrow 3 F_{c}\left(Q^{2}\right)\left[f\left(Q^{2}\right)+\kappa \tilde{f}\left(Q^{2}\right)\right] \\
G_{M}^{n}\left(Q^{2}\right) & \rightarrow-2 F_{c}\left(Q^{2}\right)\left[f\left(Q^{2}\right)+\kappa \tilde{f}\left(Q^{2}\right)\right]
\end{aligned}
$$

It is now worthwhile to make the following two observations:

- the approximation leading to Eqs. (18) and (20) is mainly based on neglecting the constituent initial transverse motion in the Melosh rotations. Thus, Eq. (18) can represent a good approximation of the full calculations (10-11) only when the average value of the transverse momenta, $\left\langle p_{\perp}\right\rangle$, is much smaller than the constituent mass $m$. However, in $Q C D$ both $m$ and $\left\langle p_{\perp}\right\rangle$ are expected to be of the order of the $Q C D$ scale, $\Lambda_{Q C D} \sim 300 \mathrm{MeV}$. Moreover, in quark potential models $\left\langle p_{\perp}\right\rangle$ turns out to be significantly larger than $m$, because of the high momentum components generated in the light-baryon wave functions by the short-range part of the effective quark-quark interaction [8];

- the Melosh rotations break in general the $S U(6)$ symmetry. Indeed, these rotations, being momentum and spin dependent, produce a re-coupling of the constituent orbital angular momentum and spin; in other words, even if the canonical wave function (6) is assumed to be factorized into a spatial part times a spin-isospin wave function (which can be classified according to the $S U(6)$ multiplets), after the application of the Melosh rotations (5) the light-front wave function (国) cannot be any more expressed as a product of a spatial part times a spin-isospin function. As a result, the light-front wave function (4) is not $S U(6)$ symmetric and therefore we do not expect in general to have $G_{E}^{n}\left(Q^{2}\right)=0$ and $G_{M}^{p}\left(Q^{2}\right) / G_{M}^{n}\left(Q^{2}\right)=-3 / 2$.

Consequently, the inclusion of the effects of the quark initial transverse motion in Eqs. (10 111) could lead to $G_{E}^{n}\left(Q^{2}\right) \neq 0$ and $G_{M}^{p}\left(Q^{2}\right) / G_{M}^{n}\left(Q^{2}\right) \neq-3 / 2$. The former point is clearly illustrated in Fig. 1, where the results of the calculations of Eqs. (10-11), performed for point-like $C Q$ 's adopting a simple gaussian-like ansätz for the radial function $w_{S}(\vec{k}, \vec{p}) \propto$ $\exp \left[-\left(k^{2}+3 p^{2} / 4\right) / 2 a_{H O}^{2}\right]$, are reported for various values of the quantity $\left\langle p_{\perp}\right\rangle=\sqrt{4 / 3} a_{H O}$ at $m=220 \mathrm{MeV}$ (chosen as in Ref. [12]). It can clearly be seen that relativistic effects may contribute significantly to $G_{E}^{n}\left(Q^{2}\right)$ when $\left\langle p_{\perp}\right\rangle>m$ [. Note that, as $\left\langle p_{\perp}\right\rangle$ increases, the neutron charge radius appears not to exceed $\sim 40 \%$ of its experimental value.

The $S U(6)$ breaking associated to the Melosh rotations heavily affects also the ratio $G_{M}^{p}\left(Q^{2}\right) / G_{M}^{n}\left(Q^{2}\right)$, as it is illustrated in Fig. 2. It should be reminded that the experimental data on $G_{M}^{p}\left(Q^{2}\right)$ and $G_{M}^{n}\left(Q^{2}\right)$ exhibit the well-known dipole behaviour, leading to $G_{M}^{p}\left(Q^{2}\right) / G_{M}^{n}\left(Q^{2}\right) \simeq \mu_{p} / \mu_{n} \simeq-1.46$ with only a $10 \div 15 \%$ uncertainty up to $Q^{2} \sim 1(\mathrm{GeV} / \mathrm{c})^{2}$

${ }^{c}$ This result does not depend on the particular choice of a gaussian-like ansätz for the radial function $w_{S}(\vec{k}, \vec{p})$, provided the average value $\left\langle p_{\perp}\right\rangle$ is kept the same. In particular, the nucleon eigenfunction corresponding to the quark potential model of Ref. [12, yields $\left\langle p_{\perp}\right\rangle \simeq 0.58 \mathrm{GeV}$; we have checked that the use of the $S U(6)$-symmetric part of this wave function leads to results almost coinciding with the solid curve of Fig. 1, which corresponds indeed to $\left\langle p_{\perp}\right\rangle \simeq 0.58 \mathrm{GeV}$. 
(cf., e.g., Ref. [1]). Thus, Figs. 1 and 2 clearly indicate that relativistic effects alone cannot explain simultaneously the experimental data on $G_{E}^{n}\left(Q^{2}\right)$ and $G_{M}^{p}\left(Q^{2}\right) / G_{M}^{n}\left(Q^{2}\right)$; therefore, other mechanisms, like, e.g., the presence of the mixed-symmetry S-wave generated by the spin-spin forces among $C Q$ 's and/or the effects of flavour-dependent $C Q$ form factors and/or the contribution of many-body e.m. currents, have to be invoked.

In conclusion, the neutron charge form factor $G_{E}^{n}\left(Q^{2}\right)$ has been investigated within a constituent quark model formulated on the light-front. It has been shown that, if the quark initial motion is neglected in the Melosh rotations, the Dirac neutron form factor $F_{1}^{n}\left(Q^{2}\right)$ receives a relativistic correction which cancels exactly against the Foldy term in $G_{E}^{n}\left(Q^{2}\right)$, as it has been recently argued in Ref. [5]. The same type of cancellation does not occur in case of the proton charge form factor $G_{E}^{p}\left(Q^{2}\right)$. Moreover, at the same level of approximation the ratio of the proton to neutron magnetic form factors $G_{M}^{p}\left(Q^{2}\right) / G_{M}^{n}\left(Q^{2}\right)$ is still given by the naive $S U(6)$-symmetry expectation, $-3 / 2$. These results are independent of the electromagnetic structure of the constituents, provided their form factors are $S U(2)$ symmetric. However, since the full Melosh rotations break $S U(6)$ symmetry, both $G_{E}^{n}\left(Q^{2}\right) \neq$ 0 and $G_{M}^{p}\left(Q^{2}\right) / G_{M}^{n}\left(Q^{2}\right) \neq-3 / 2$ can be obtained even when a $S U(6)$-symmetric canonical wave function is assumed. It has been shown that relativistic effects alone cannot explain simultaneously the experimental data on $G_{E}^{n}\left(Q^{2}\right)$ and $G_{M}^{p}\left(Q^{2}\right) / G_{M}^{n}\left(Q^{2}\right)$.

Acknowledgements. One of the authors, S.S., is deeply indebted with Nathan Isgur for many fruitful discussions while at the Institute for Nuclear Theory in Seattle, where part of the present work has been carried out. The warm hospitality and the high-level scientific environment found at the INT during the Int'l Workshop on Algebraic Methods in Many Body Physics, organised by Franco Iachello and Joseph Ginocchio, are also gratefully acknowledged. 


\section{References}

[1] See, for a recent review, G. Petratos: in Proc. of the Int'l Workshop on The Structure of the Nucleon, INFN National Laboratory of Frascati (Italy), June 7-9, 1999, to appear in Nucl. Phys. A.

[2] R.G. Sachs: Phys. Rev. 126 (1962) 2256.

[3] S. Kopecki et al.: Phys. Rev. Lett. 74 (1995) 2427.

[4] See, e.g., T. Ericson and W. Weise: Pions and Nuclei, Claredon Press (Oxford, 1988).

[5] N. Isgur: Phys. Rev. Lett. 83 (1999) 272.

[6] P.L. Chung and F. Coester: Phys. Rev. D44 (1991) 229.

[7] F. Cardarelli et al.: Phys. Lett. B357 (1995) 267; Few Body Syst. Suppl. 8 (1995) 345.

[8] F. Cardarelli et al.: Few Body Syst. Suppl. 11 (1999) 66.

[9] L.L. Frankfurt and M.I. Strikman: Nucl. Phys. B 148 (1979) 107. G.P. Lepage and S.J. Brodsky: Phys. Rev. D22 (1980) 2157. S.J. Brodsky and G.P. Lepage: in Perturbative Quantum Chromodynamics, edited by A.H. Mueller, World Scientific (Singapore, 1989), p. 93-240. T. Frederico and G.A. Miller: Phys. Rev. D45 (1992) 4207. M. Sawicki: Phys. Rev. D46 (1992) 474.

[10] F. Cardarelli: PhD thesis, University of Rome "La Sapienza", 1995, unpublished.

[11] F. Cardarelli and S. Simula: Phys. Lett. B421 (1998) 295 and e-print archive hepph/9810414, to appear in Phys. Rev. D.

[12] S. Capstick and N. Isgur: Phys. Rev. D34 (1986) 2809.

[13] S. Platchkov et al.: Nucl. Phys. A510 (1990) 740. 


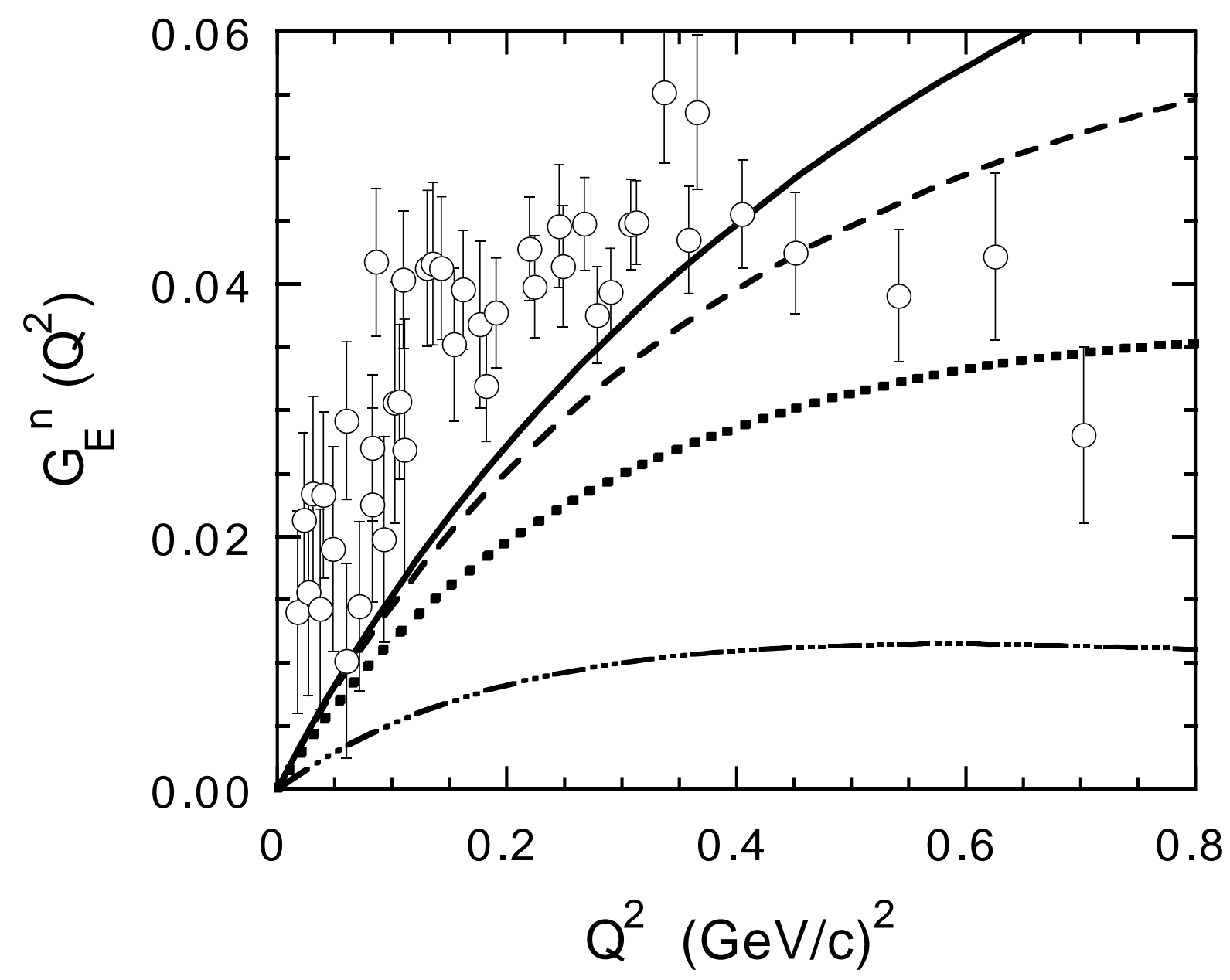

Figure 1. The neutron charge form factor $G_{E}^{n}\left(Q^{2}\right)$ versus $Q^{2}$. The experimental data correspond to the results of the analysis of Ref. [13] performed in terms of the Reid-Soft-Core nucleon-nucleon interaction. The various curves are the results of the calculations of Eqs. (10-11), obtained assuming point-like $C Q$ 's and adopting a simple gaussian-like ansätz for the radial function $w_{S}(\vec{k}, \vec{p})$ (see text). The value of the constituent quark mass has been chosen to be $m=220 \mathrm{MeV}$ from Ref. [12]. The dot-dashed, dotted, dashed and solid lines correspond to $\left\langle p_{\perp}\right\rangle=\sqrt{4 / 3} a_{H O}=0.23,0.35,0.46$ and $0.58 \mathrm{GeV}$, respectively. 


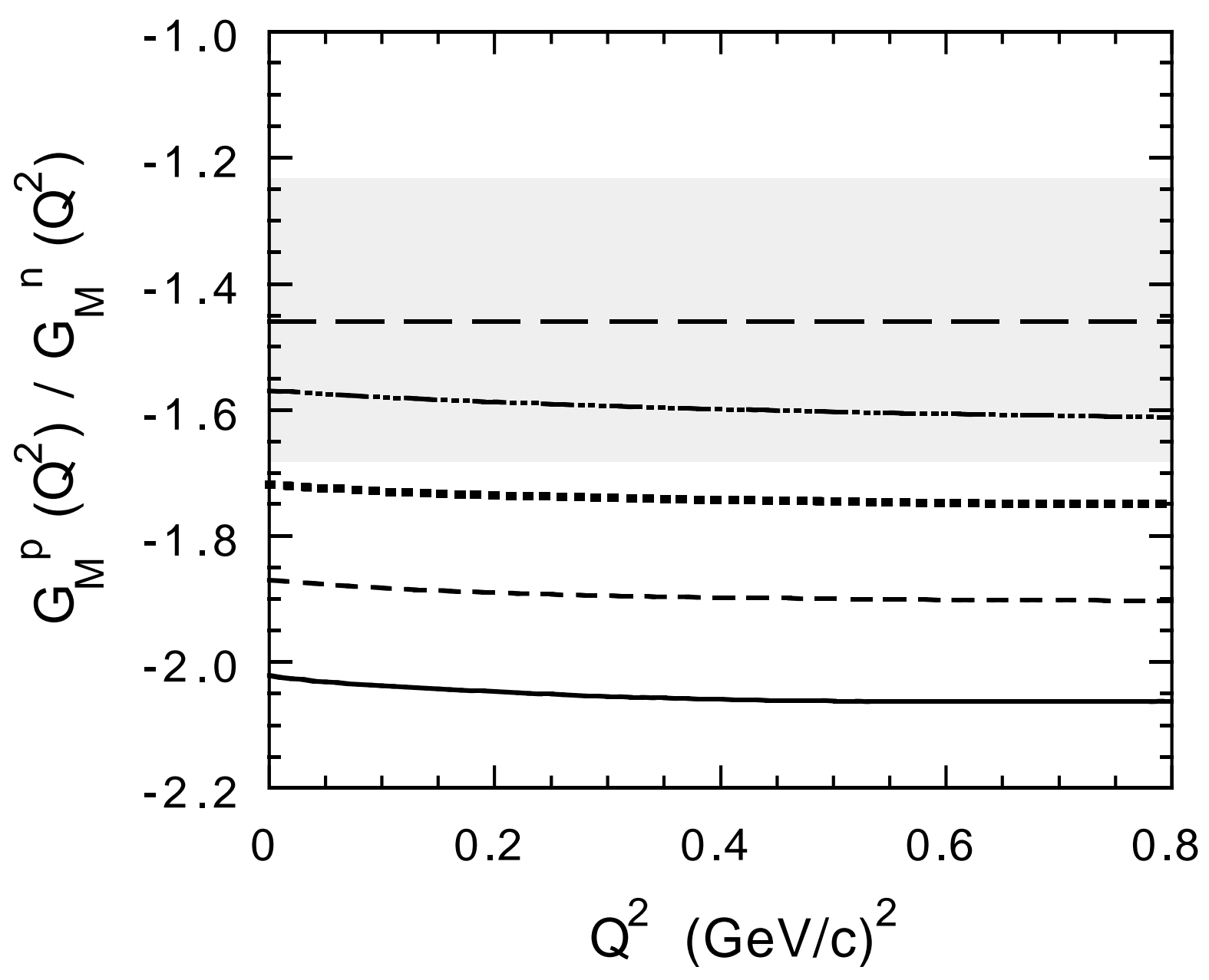

Figure 2. The ratio of the proton to neutron magnetic form factors $G_{M}^{p}\left(Q^{2}\right) / G_{M}^{n}\left(Q^{2}\right)$ versus $Q^{2}$. The shaded area corresponds to a $\pm 15 \%$ deviation from the dipole-fit expectation $G_{M}^{p}\left(Q^{2}\right) / G_{M}^{n}\left(Q^{2}\right) \simeq \mu_{p} / \mu_{n} \simeq-1.46$ (long-dashed line). The dot-dashed, dotted, dashed and solid lines are as in Fig. 1. 